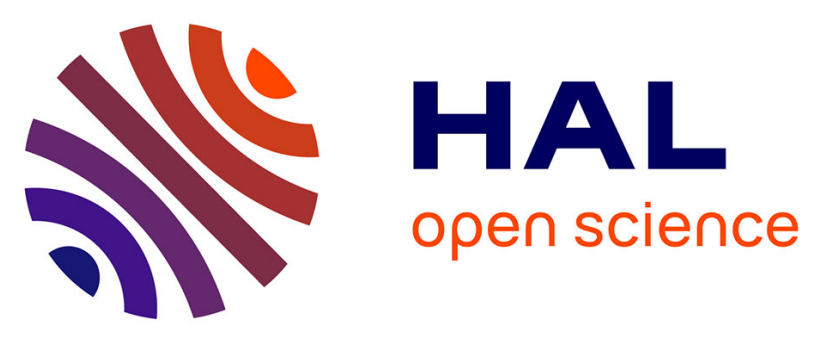

\title{
Non-conventional imaging systems for 3D digitization of transparent objects: Shape from polarization in the IR and shape from visible fluorescence induced UV
}

Fabrice Meriaudeau, Rindra Rantoson, Kedir Adal, David Fofi, Christophe Stolz

\section{To cite this version:}

Fabrice Meriaudeau, Rindra Rantoson, Kedir Adal, David Fofi, Christophe Stolz. Non-conventional imaging systems for 3D digitization of transparent objects: Shape from polarization in the IR and shape from visible fluorescence induced UV. 3RD INTERNATIONAL TOPICAL MEETING ON OPTICAL SENSING AND ARTIFICIAL VISION: OSAV'2012., May 2012, Saint Pétersbourg, Russia. pp.34-40, 10.1063/1.4809689 . hal-00860078

\section{HAL Id: hal-00860078 https://hal.science/hal-00860078}

Submitted on 10 Sep 2013

HAL is a multi-disciplinary open access archive for the deposit and dissemination of scientific research documents, whether they are published or not. The documents may come from teaching and research institutions in France or abroad, or from public or private research centers.
L'archive ouverte pluridisciplinaire HAL, est destinée au dépôt et à la diffusion de documents scientifiques de niveau recherche, publiés ou non, émanant des établissements d'enseignement et de recherche français ou étrangers, des laboratoires publics ou privés. 


\title{
Non Conventional Imaging Systems for 3D Digitization of Transparent Objects. Shape from polarization in the IR and shape from visible Fluorescence induced UV.
}

\author{
F. Meriaudeau, R. Rantoson, K.M. Adal, D. Fofi C. Stolz \\ Université de Bourgogne, Le2i, UMR 6306, 12 rue de la fonderie - 71200 Le Creusot - France \\ E-mail:fmeriau@u-bourgogne.fr
}

\begin{abstract}
This paper presents a comparison between recent advances made in the field of non conventionnal imaging techniques for 3D digitization of transparent object. After a large survey, this paper will focus on two recent techniques later called : shape from Visible Fluorescence UV-induced and shape from polarisation in the IR which recently emerged. Results obtained with the technique of Scanning from Heating which, originaly developed in 2008 for the digitization of transparent objects, has sucessfully been modified and applied to the digitization of specular objects.
\end{abstract}

Keywords: 3D Digitization, transparent object, shape from polarization.

PACS: 06.20.-f, 07.05.P, 07.60.-j

\section{INTRODUCTION}

Machine vision systems which appear in the industry 25 years ago have greatly benefit from the sensor evolution (resolution as well as spectral band) and from the computing resources. Nowadays, systems include a large amount of information such as multispectral or color information, polarization information, associated with high level image processing or classification schemes. Meanwhile, the marketing and the fashion is leading to a production of complex products, creating the need to develop new systems able to recover and control the $3 \mathrm{D}$ aspects of complex ('in shape and in composition) objects. 3D scanning has been investigated for several decades and most of the proposed approaches assume a diffuse or near diffuse reflectance of the object's surface. The broad literature on the subject is usually divided into active and passive techniques. Active light techniques, whose a recent review is proposed by Blais [2], include laser range scanning, coded structured light systems, digital holography or time-offlight scanners whereas passive techniques are mainly stereovision, photogrammetry or shape from $\mathrm{X}$ techniques (shading, optical flow, motion, focus...).

For transparent objects and in most cases for mirror-like surfaces, a thin layer of powder is generally sprayed onto the object (to make its surface opaque and diffuse) prior to its digitization. This extra step is troublesome, time consuming (the object needs to be cleaned afterwards), the final accuracy is often dependent on the powder thickness and its homogeneousness and in the case of defect detection, these later one can be overlooked and missed in the final classification step.

To overcome this procedure, various methods have been investigated over the last few years and an exhaustive and recent review can be found in [1] which was further recently completed by Meriaudeau and al. [6] for the case of transparent objects. Most of the presented methods require some a priori about the object or assumptions about the interactions of the light with the object surface and are not yet ready to be implemented in the industry. In its review, Irkhe [1] used a taxonomy of 9 object classes (from a rough surface exhibiting a diffuse or near diffuse reflectance, to a more complex surface composed of scattering, absorption, reflexion .. with full global light transport) based on increasing complexity in light transport to be categorized. Our paper proposes to include recent publications posterior to [1] and [2], including shape from from Visible Fluorescence UV-induced [3] as well as shape from polarization [4] with a recent extension in the IR [5], to compare them to the most relevant presented in [1], [2] and to scanning from heating [7], [8], which recently proved to be versatile and useable for specular object [8].

The rest of this paper is organized as follows: the first part will present the a state of the art of the techniques which lead to the 3D geometry of a transparent objects, the second part will focus on two methods which we 
recently investigated : shape from polarisation in the IR and shape from Visible Fluorescence UV-induced. The last part will attempt to classify and compare these methods regarding their potential applicability within an industrial context of quality control by optical inspection for which processing time, accuracy, cost and applicability (in term of versatility) are of high importance.

\section{STATE OF THE ART}

This paper aims at presenting systems/methods which could lead to machine vision devices and do not therefore put any emphasis on metrology systems such as white light interferometry and deflectometry which both have proven to give high quality results for object with a simple (free form) surface for curvature inspection but are too restrictive for complex objects.

It has to be noted, that, as highlighted by the survey of Irkhe [1] some of the techniques, which for most were inspired from the computer graphics community, are quite versatile and can be used, with the correct $a$ priori, for transparent as well as for specular objects.

Kutulakos and Stegger [10] proposed in 2005, a nice approach where the main idea is to seek to invert the indirect projection process. Knowing the background or the environment (also called environment matting), some a priori about the object (refraction index) and subject to certain restrictions: number of viewpoints higher than three, number of reflexions/refractions limited to two... they were able to achieve the 3D reconstruction of simple transparent objects can be reconstructed. Only one object was reconstructed (diamond shape) with a fair accuracy.

Two years later, Kutulakos and Morris [11] proposed an efficient method based on scatter-trace photography. Their approach involves capturing images of the scene from one or more viewpoints while moving a proximal light source to a $2 \mathrm{D}$ or $3 \mathrm{D}$ set of positions. This gives a $2 \mathrm{D}$ (or 3D) dataset per pixel, called the scatter trace. The scatter trace of each pixel has a highly constrained geometry that (1) reveals the contribution of direct surface reflection, and (2) leads to a simple "scatter trace stereo" algorithm for computing the local geometry of the exterior surface (depth and surface normals). This technique provides reasonable results (accuracy of few mm RMS were reported) for objects with an inhomogeneous interior, but with rather simple shape (smooth surface). Another limitation of this technique is the processing time. A recent low cost version [12], which results offer perspective for computer graphics has just been published..

A very similar approach, based on inverse ray tracing was recently presented by Iwabuci [13] et al. Their technique employs several sensors placed around a simple transparent object (no complex interior) and can infer the shape as well as the index of refraction of the object.

In the same period, Trifonov et al.[14] estimated the shape of a transparent object through visible tomography. Their technique requires immersing in a liquid the object to be digitized. The fact that the index of refraction of the object and the liquid have to be identical to avoid spurious inter-reflections and refractions between the fluid and the object, is very restrictive and is not suited for any transparent object with opaque cavities inside it.

All these techniques rely on the principle of a analyzing the transmitted wave. Similarly, methods which infer the 3D shape while exploiting the reflecting beam are thereafter presented.

Skydan et al. [15] proposed a method close to "deflectometry" to analyze the shape of simple convex shape of transparent object (automotive glass). Their method is quite simple, but the surface height recovery is quite dependant on pre-processing steps as well as on the phase unwarping algorithm.

Hullin et al.[16] proposed a technique borrowed from the structured light technique. The objects are immersed in a fluorescent liquid and illuminated with a laser sheet, resulting in black stripes at the object edges (where fluorescence doesn't occur) and used for triangulation. Complex objects can be reconstructed; but no error maps are provided. Moreover the technique is too restrictive (restricted size of the object, the need for immersing the object as well as the need for matching the index of refraction of the object with the one of the liquid) to be used in an industrial context.

Yamazaki et al. [17] associated Stereo and Phase shift methods to recover the shape of simple transparent objects by recovering the front and back surfaces of the object, therefore restricting the approach to only two refractions/reflexions onto the objects. The technique also requires an active lighting system which, as for environment matting, has to be moved to several positions near the object and is therefore not suitable for industrial environment.

Myasaki and coworkers [18-20] developed a technique based on "shape from polarization" which was later successfully applied to metallic specular surfaces [21]. This technique will be described in the next part with an extension in the IR region. 
More recently, Klank et al. [22] developed a system using active Infrared illumination. The principle relies on the fact that transparent object made of glass absorb the infrared radiation whereas the background will reflect it. Therefore a Time of Flight Camera sensitive to these wavelengths can infer the 3D geometry of the foreground transparent objects. Primary results showed a proof a concept but should be further investigated prior to be deployed within the industry, then can however be used for obstacles detection in robot navigation.

Recently Eren [23] and Meriaudeau [24] proposed a technique called "scanning from heating" (SFH) where an IR pattern is simply projected onto the object generating heat which is then released by the object and imaged by a spatially calibrated IR sensor; the technique relies, therefore, on the observation of the emitted pattern which can be a dot or a line. Various objects were scanned and an industrial prototype is currently being used in Turkey. Authors obtained accurate results (errors between $200 \mu \mathrm{m}$ ), with deviation maps calculated between their model and those obtained with a Minolta VI590 scanner after coating the object to make it diffuse This technique is highly reliable, versatile (an extension for specular object was recently being made [25]) but relies on costly equipments. (one or two IR cameras visualizing the heat released pattern as well as an IR laser).

Among the described technique the Scanning from Heating is the best one for an implementation within the industry aiming at controlling various transparent objects made of different material. However its high price has led researcher to develop complementary techniques at a lower cost which will be depicted in the next part.

\section{RECENT NON CONVENTIONAL METHODS}

\section{Shape from Visible Fluorescence UV- Induced}

Our approach [3] proposes a non contact measurement system for transparent objects which solves the refraction problems by mean of a UV structured light in a triangulation scheme. The novelty of the proposed approach lies in the exploitation of the fluorescence generated at the object surface under the irradiation of a UV Laser using a specific triangulation approach associated with a fluorescence points tracking method. The approach is extendable and adaptable for industrial applications such as glass inspection in quality control and 3D modelling of transparent objects. Two experimental configuration are presented (see FIGURE 1), the first one relies on a point projection whereas the other on is an extension of the former with a line generated by a hemi-cylindrical lens. Results on more than 10 different objects of various shapes, various materials (glass, plastic...), various thicknesses were reported [3]. The achieved mean deviation error can be as low as $100 \mu \mathrm{m}$ showing the high potential of the method.

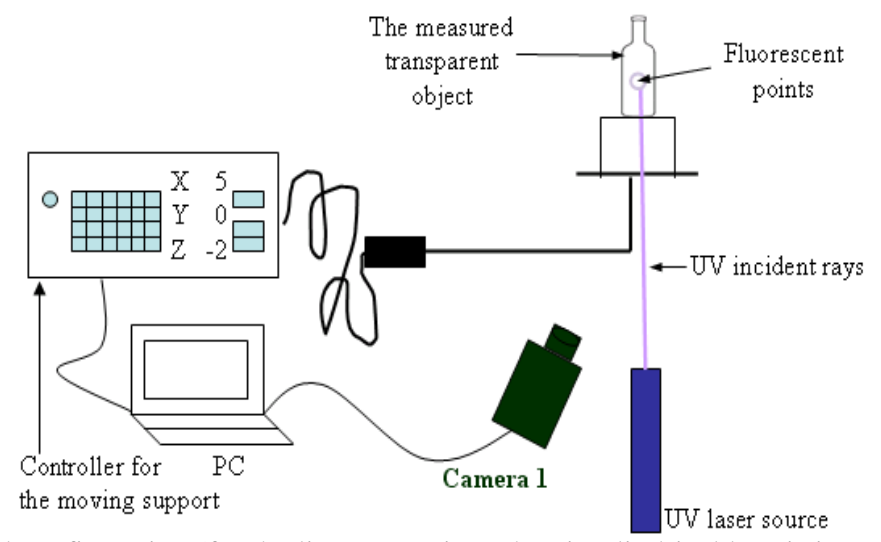

FIGURE 1. a) Experimental configuration (for the line generation a hemi-cylindrical lens is inserted between the laser and the object. 


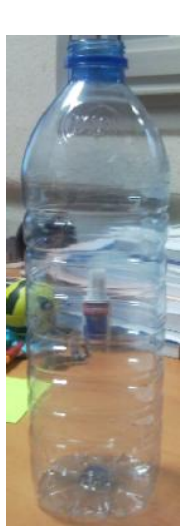

a )

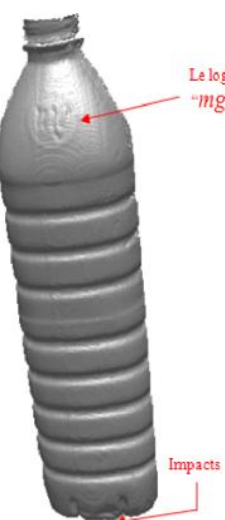

b)

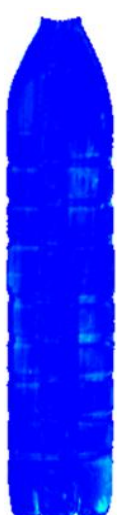

average

Total distribution:

$99.99381 \%$

$0.000000 .80000 \quad 2.00000$

c)

FIGURE 2. a) Original plastic bottle b) plastic bottle digitized with our method c) associated error map (mean error $80 \mu \mathrm{m}$, standard deviation $90 \mu \mathrm{m}$ )
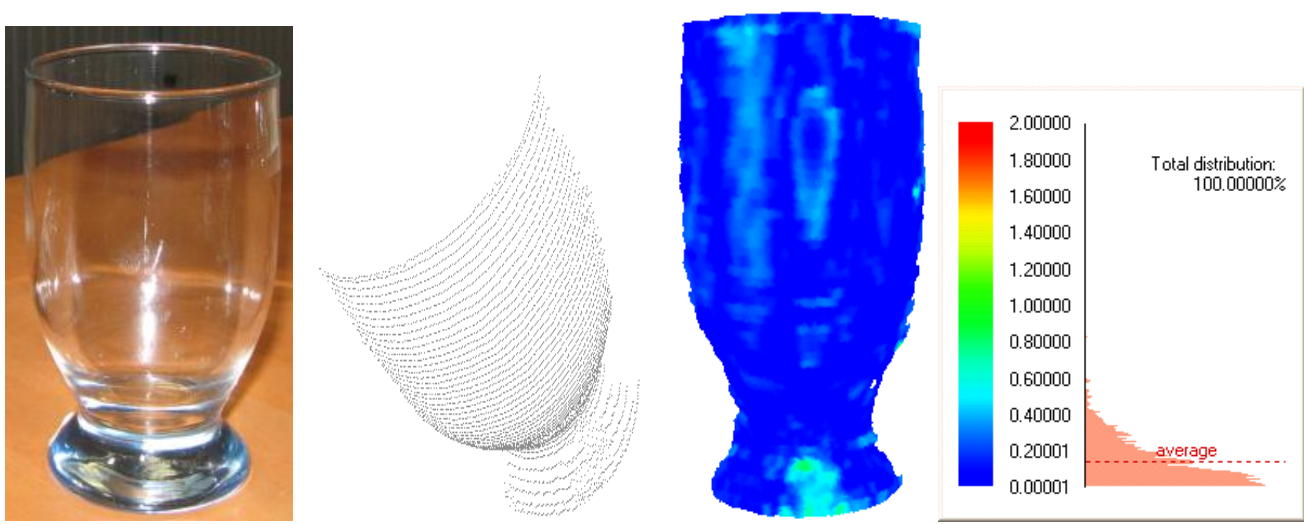

FIGURE 3. a) Original glass b) 3D reconstruction obtained with line projection c) associated error map (mean error $140 \mu \mathrm{m}$, standard deviation $120 \mu \mathrm{m})$

\title{
Shape from Polarization in the IR
}

\author{
Shape from Polarization : Principle
}

Starting from the work of Wolff [26], [27] in the early 1990s who designed a polarimetric camera with applications dedicated to material identification and separation of diffuse and specular components in the observed scene, Rahmann [28], [29] and Saito [30] showed the possibility to link polarimetric parameters to depth in the observed scene leading to the new concept of "shape from polarization". The aim of the shape from polarization technique is to measure the normal at each point and then to obtain the whole surface by integration of the normals field.

The shape from polarization was applied by Miyasaki et al [31], [32], [33] and Ferraton and al. [34] for transparent objects and by Morel et al. [35] for their metallic counter parts. Whereas Ferraton and al. [34] proposed a multispectral approach combined with an active lighting to solve the ambiguities on the azimuthal and zenithal angles, Miyasaki [32] was the first one to consider the use of IR combined (two different set-up) with the visible. Unfortunately, the former technique requires a very minute calibration and is quite sensitive to temperature changes preventing it from being used in the industry as such, and the later one needs two different set-ups, one in the visible and one in the IR, implying either registration or a cumbersome optical system. To overcome these two problems, we propose an active shape from polarization in the IR which will be fully described in the next section. 
The principle of polarization imaging relies on the following principle: after reflection, a non-polarized light wave becomes partially linearly polarized according to the normal to the surface and the optical index of the material at the point of incidence. A partially polarized wave may be defined by three parameters which are: the light intensity I, the degree of polarization $\rho$ and angle of polarization $\varphi$. The two latest parameters are respectively linked to zenithal angle $\theta$ and the azimuthal angle $\phi$ through the Snell-Descartes relations. Therefore measuring the degree of polarization $\rho$ and angle of polarization $\varphi$ enables to infer the normal at each point by means of the zenithal angle $\theta$ and the azimuthal angle $\phi$. This normal field is then integrated to recover the 3D shape of the object.

The study of the state of polarization of a light wave requires a rotating polarizer placed in front of an orthographic camera or a combination of active components acting as a rotating polarizer [36]. The relationship (see Figure. 3) between the measured intensity Ip, the total light magnitude $\mathrm{I}_{\text {tot }}$, the polarization angle $\varphi$ and the orientation angle $\alpha$ of the polarization filter is given by:

$$
I_{p}(\alpha)=\frac{I_{t o t}}{2}(\rho \cos (2 \alpha-2 \varphi)+1)
$$

Where, the degree of polarization is defined by

$$
\rho=\frac{I_{\max }-I_{\min }}{I_{\max }+I_{\min }}
$$

And it is equal to zero when the light is not polarized and equal to 1 when the light is linearly polarized which corresponds to the incident angle equals to the Brewster angle.

Equation (1) can also be written as:

$$
I_{p}(\alpha)=\frac{1}{2}\left(S_{0}+S_{1} \cos 2 \alpha+S_{2} \sin 2 \alpha\right)
$$

Where $S_{0}, S_{1}$ and $S_{2}$ are the unknown Stokes parameters [39] expressed as:

$$
\begin{aligned}
& S_{0}=I_{t o t} \\
& S_{1}=\rho I_{t o t} \cos 2 \varphi \\
& S_{2}=\rho I_{t o t} \sin 2 \varphi
\end{aligned}
$$

The purpose of the polarization imaging is to calculate $I_{\text {tot }}, \rho$ and $\varphi$ through formulas (1) and (2) or (3) and (4). We used the second solution, by taking 18 images with the polarizer turned of $10^{\circ}$ between each acquisition, and solve the system using least mean squares. This solution is more robust against noise compared to the evaluation of the parameters through equations (1) and (2).

Admissibility of the Stokes vector was also investigated prior to any reconstruction, enabling to remove noisy data points [5].

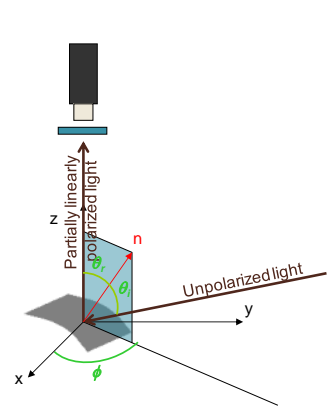

a)

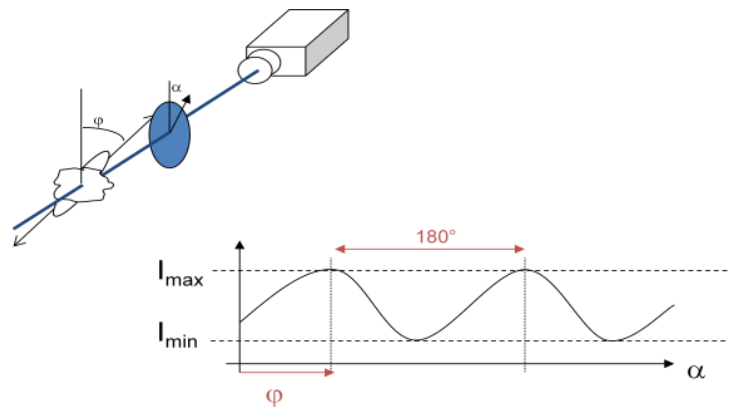

b) c)

FIGURE 3. Principle of the polarization measurements: a) principle of the shape from polarization, b) optical set-up and c) intensity transmitted through the polarizer.

However, even for the Brewster angle, the degree of polarization is less than 1, this can be compensated by introducing a complex index, where $\mathrm{n}$ is the real index and $\mathrm{k}$ the coefficient of extinction of the material being studied. This value has to be estimated empirically for each studied sample. 
As pointed out earlier by Myasaki [31-33] and Morel [35], the measures of degree of polarization $\rho$ and of the angle of polarization $\varphi$ do not allow a straightforward evaluation of the normal vector defined by zenithal angle $\theta$ and azimuthal angle $\phi$.

Indeed, both the degree of polarization $\rho$ and the angle of polarization $\varphi$ provide two candidates for each measure.

To remove the ambiguity on $\phi$ and select the right candidate, we used an active lighting system based on Morel approach [16]. Working on the far IR range shifts the value of the Brewster angle to high values, enabling to infer the correct value of the zenithal angle $\theta$ from the degree of polarization for surface with angle ranging from 0 to $73^{\circ}$ (see Figure 4)

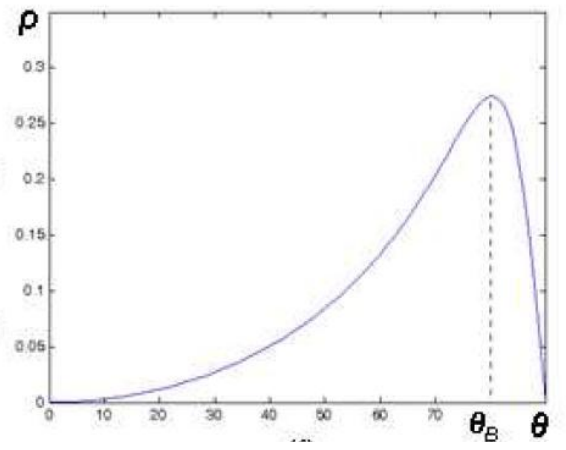

FIGURE 4. Evolution of the degree of polarization versus the zenithal angle in IR band.

\section{Experimental Set Up and Results}

Our IR imaging system (Figure.5 (a)) is composed of a thermal camera FLIR SC-645 IR of [7.5 $\mu \mathrm{m}-13 \mu \mathrm{m}]$ range, of $640 \times 480$ resolution, $24.5 \mathrm{~mm}$ of non telecentric focal lens; a manual rotating ( $\mathrm{ZnSe}$ ) polarizer of [1 $\mu \mathrm{m}-15 \mu \mathrm{m}]$ range with an orientation angle $\alpha$; a specific dome (generating the quadrant active IR lighting) composed of two pieces: a metallic cover which top is holed to place the camera, and a slab of 56 resistors (Figure. 5 (b)) (12 Ohms and $0.25 \mathrm{~W}$ each) supplied by a voltage of $12 \mathrm{~V}$ for each quadrant illumination. The temperature of each resistor is constant $\sim 60$ degrees Celsius during the acquisitions, corresponding to a maximum radiation of $8.7 \mu \mathrm{m}$ according to Wien's law. The camera calibration is done with an IR checkerboard made of two different materials of two different emissivites (Figure 5 (c)) using Bouguet ${ }^{\circledR}$ Toolbox. The calibration, enables us estimating $\mathrm{Z}_{0}$, the distance between the object support and the camera [38] to take into account the fact that a perspective camera is used instead of an orthographic one. An example of a reconstructed object is presented on figure 5.(d).

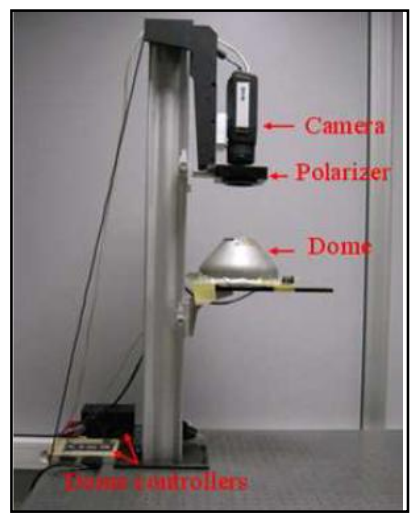

a)

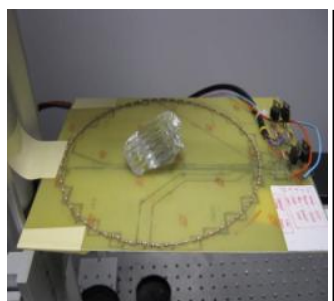

b)

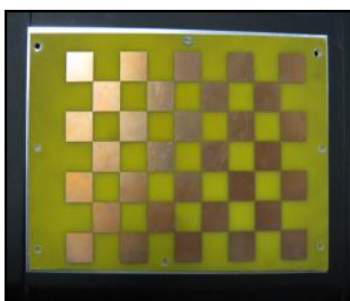

c)

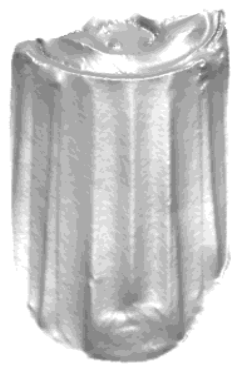

d)

FIGURE 5: a) Experimental Set-Up, b) lighting system in the IR made of resistors and which can be turned on by quadrants c) The checkerboard made of two materials of different emissivity for use in IR, d) reconstruction of a glass sample 


\section{CONCLUSION}

This paper presents a survey about recent techniques for the digitization of transparent objects. All techniques were briefly recalled and compared with each other, with some highlights on their respective advantages and drawbacks (see table 1).

The shape from fluorescence is from far the best tradeoff between price and accuracy and has even shown possible extension for digitization of specular objects. Its only disadvantage is the fact that some parameters need to be tuned for different material compositions. The scanning from heating with a recent extension to the digitization of specular surfaces is also an interesting technique which only drawback is its high price due to the use of IR cameras. The immersion fluorescence technique, provides accurate results but suffers from the supplementary steps of immersing the product which is quite similar as coating them with a powder prior to a digitization with a commercial 3D scanner.

The shape from polarization has also a high accuracy and its recent extension to the IR spectrum shows some new potentiality. A scanning device which will merge all these techniques would also be interesting to investigate in the near future.

TABLE 1 : Comparison of the various method

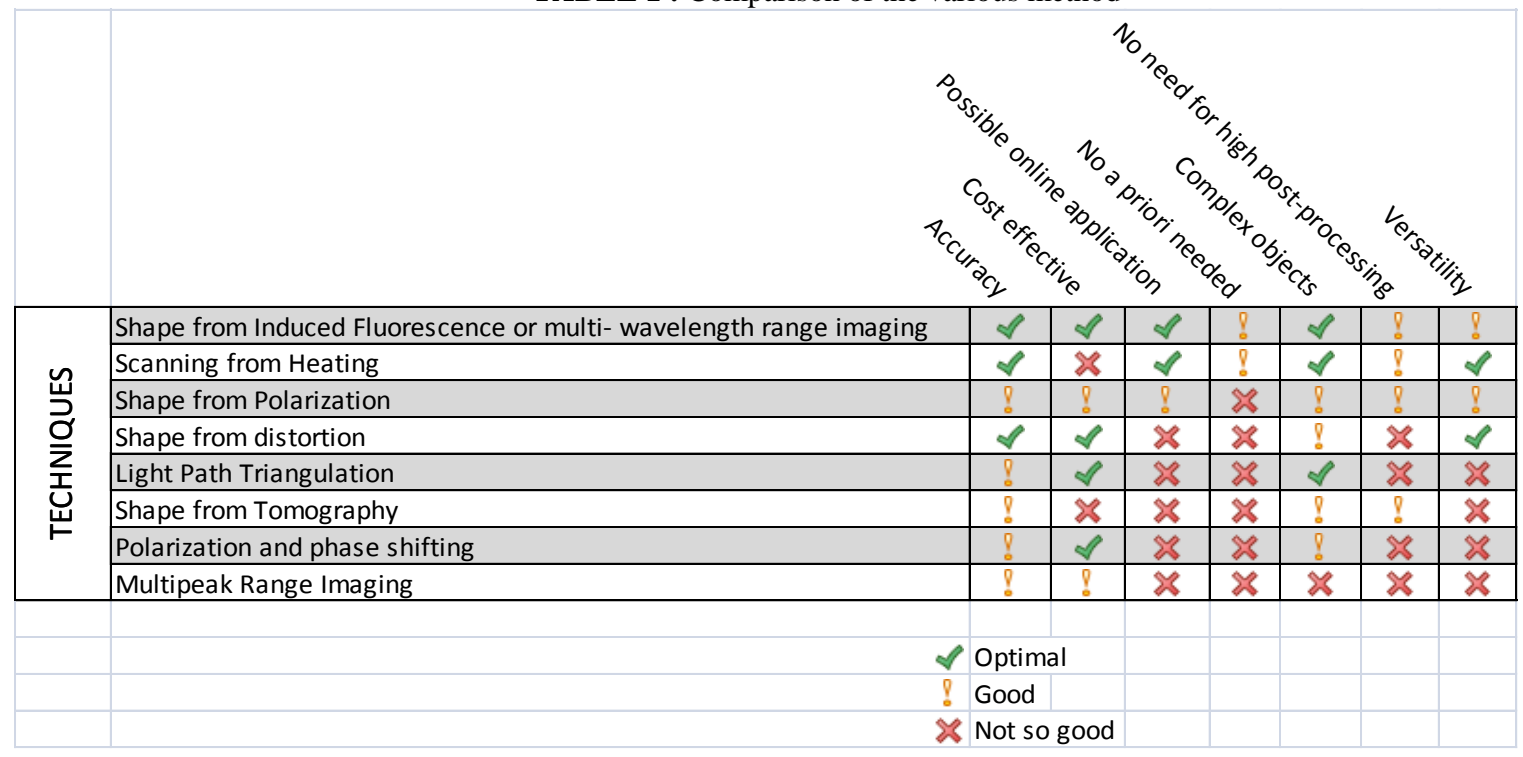

\section{REFERENCES}

1. I. Ihrke, Kiriakos N. Kutulakos, Hendrik P. A. Lensch, Marcus Magnor, Wolfgang Heidrich , Transparent and Specular Object Reconstruction, Computer Graphics Forum, Vol. 29, number 8 pp. 2400-2426, (2010).

2. F. Blais, Review of 20 Years of Range Sensor Development, Journal of Electronic Imaging 13, 1,pp. (231-243, (2004).

3. R. Rantoson, C. Stolz, D. Fofi and F. Meriaudeau "Optimization of Transparent Objects Digitization from Visible Fluorescence UV-induced", , Optical Engineering 51(3), 033601 (March 2012).

4. M. Ferraton, C. Stolz, F. Meriaudeau "Optimization of a polarization imaging system for 3D measurements of transparent objects", Optics Express, Optical Society of America, 17 (23), pp. 21077-21082, (2009).

5. R. Rantoson, "Numérisation 3D d'Objets Transparents par Polarisation dans l'IR \& par Triangulation dans l'UV », PhD dissertation, Burgundy University, (November 2011).

6. "Review and comparison of Non Conventional Imaging Systems for 3D Digitization of transparent objects", F. Mériaudeau, R. Rantoson, D. Fofi, C. Stolz, Journal of Electronic Imaging, 21, 2(2012) 021105, 2012.

7. F. Meriaudeau, L.A. Sanchez Secades, G. eren, A. Erçil, F. Truchetet, O. Aubreton, D. Fofi, David Fofi "3D Scanning of Non-Opaque Objects by means of Imaging Emitted Structured Infrared Patterns ", IEEE Transactions on Instrumentation \& Measurement, Vol 59, №11, pp 2898 2906, (2010). 
8. Gonen Eren, Olivier Aubreton, Fabrice Meriaudeau, Luis Alonzo Sanchez-secades, David Fofi, Frédéric Truchetet, Aytul Erçil "Scanning From Heating: 3D Shape Estimation of Transparent Objects from Local Surface Heating, Optics Express, Optical Society of America, 17 (14), pp. 11457-11468, 2009.

9. A.Bajard, O.Aubreton, Y.Bokhabrine, B.Verney, G.Eren, A.Erçil, F.Truchetet "3D Scanning of specular and diffuse metallic surfaces using an infrared technique", , Accepted in Optical Engineering, 2012.

10. K.N. Kutulakos, E. Steger "A theory of refractive and specular 3D shape by Light-Path Triangulation, , in IEEE ICCV, pp 1448-1455, (2005).

11. N. J. W. Morris and K. N. Kutulakos, reconstructing the surface of inhomogeneous transparent scenes by scatter-trace photography, in Proc. IEEE 11th ICCV, pp. 1-8, (2007).

12. Sai-Kit Yeung, Tai-Pang Wu, Chi-Keung Tang, Tony F. Chan and Stanley Osher "Adequate Reconstruction of Transparent Objects on a Shoestring Budget", IEEE Conference on Computer Vision and Pattern Recognition, (2011).

13. Y. Iwabuchi, L. Li,M. Baba and K. Ohtani, "3D shape measurement of a transparent object with unknown refractive index by inverse ray tracing method", SICE Annual Conference 2011 September 13-18, 2011, Waseda University, Tokyo, Japan, (2011).

14. B.Trifonov, D. Bradley, W. Heidrich, Tomographic Reconstruction of Transparent Objects, Eurographics Symposium on Rendering, Tomas Akenine-Möller and Wolfgang Heidrich (Editors), (2006).

15. O A Skydan, M J Lalor and D R Burton, "3D shape measurement of automotive glass by using a fringe reflection technique", Meas. Sci. Technol. 18 , pp 106-114, (2006).

16. M. B. Hullin, M. Fuchs, I. Ihrke, H.-P. Seidel, and H. P. A. Lensch, , Fluorescent immersion range scanning, in ACM Trans. Graphics, vol. 27, no. 3, pp. 87:1-87:10, (2008).

17. M. Yamazaki, S. Iwata, and G. XuIn "Dense 3D Reconstruction of Specular and Transparent Objects Using Stereo Cameras and Phase-Shift Method”, , Y. Yagi et al. (Eds.): ACCV 2007, Part II, LNCS 4844, pp. 570-579, (2007).

18. D. Miyazaki, M. Saito, Y. Sato, and K. Ikeuchi, "Determining Surface Orientations of Transparent Objects Based on Polarization Degrees in Visible and Infrared Wavelength," J. Opt. Soc. Am. A, 19, (4), pp. 687-694, (2002).

19. D. Miyazaki, M. Kagesawa, and K. Ikeuchi, "Transparent Surface Modeling from a Pair of Polarization Images," IEEE Trans. Pattern Analysis and Machine Intelligence 26(1), 73-82, (2004).

20. M. Saito, Y. Sato, K. Ikeuchi, and H. Kashiwagi, "Measurement of Surface Orientations of Transparent Objects by Use of Polarization in Highlight", J. Opt. Soc. Am. A, 16, (9), pp. 2286-2293, (1999).

21. O. Morel, C. Stolz, F. Meriaudeau, P. Gorria "Active lighting applied to three-dimensional reconstruction of specular metallic surfaces by polarization imaging”, Appl. Opt, 45 (17), pp. 4062-4068, (2006).

22. U. Klank, D. Carton, M. Beetz, "Transparent Object Detection and Reconstruction on a Mobile Platform", IEEE International Conference on Robotics and Automation (ICRA), Shanghai International Conference Center, May 9-13, Shanghai, China, (2011).

23. G. Eren, O. Aubreton, F. Meriaudeau, L. A. Sanchez Secades, D. Fofi, F. Truchetet, and A. Erçil , "Scanning from heating: 3D shape estimation of transparent objects from local surface heating," Opt. Express, vol. 17, no. 14, pp. 11 457-11 468, (2009).

24. F. Meriaudeau, L.A. Sanchez-Secades, B. G. Eren, B. A. Erçil, F. Truchetet, A. O. Aubreton, and A. D. Fofi, “3D Scanning of Non-Opaque Objects by means of Infrared Imaging”, in IEEE On Transaction on Instrumentation and Measurement, 59 (11), pp. 2898-2906, (2010).

25. A. Bajard; O. Aubreton,G. Eren, P. Sallamand, F. Truchetet, "3D digitization of metallic specular surfaces using scanning from heating approach", Three-Dimensional Imaging, Interaction, and Measurement. Edited by Beraldin, J. Angelo; Cheok, Geraldine S.; McCarthy, Michael B.; Neuschaefer-Rube, Ulrich; Baskurt, Atilla M.; McDowall, Ian E.; Dolinsky, Margaret. Proceedings of the SPIE, Volume 7864, pp. 786413-786413-7 (2011).

26. L. B. Wolff, "Polarization vision: a new sensory approach to image understanding," Image and Vision computing, 15, pp. 8193, (1997).

27. L. B. Wolff, "Polarization-based material classification from specular reflection," IEEE Trans. On Pattern Analysis and Machine Intelligence, 12, pp. 1059-1071,( 1990).

28. S. Rahmann, "Polarization images: a geometric interpretation for shape analysis," in IEEE International Conference on Pattern Recognition (ICPR), pp. 542-546, (2000).

29. S. Rahmann, "Reconstruction of Quadrics from Two Polarization Views," in Iberian Conference on Pattern Recognition and Image Analysis (IbPRIA03), (Springer, 2003), pp. 810-820, (2003).

30. M. Saito, Y. Sato, K. Ikeuchi, and H. Kashiwagi, "Measurement of Surface Orientations of Transparent Objects Using Polarization in Highlight," in IEEE Conference on Computer Vision and Pattern Recognition, pp. 381 - 386, (1999).

31. D. Miyazaki, M. Kagesawa, and K. Ikeuchi, "Determining Shapes of Transparent Objects from Two Polarization Images," in IAPR, pp. 26-31, (2002).

32. D. Miyazaki, M. Saito, Y. Sato, and K. Ikecuhi, "Determining Surface Orientations of transparent objects on polarization degrees in visible and infrared wavelength," J. Opt. Soc. Am. A, Vol. 19, pp. 687-694, (2002).

33. D. Miyazaki, D. Miyazaki, and K. Ikeuchi, "Shape Estimation of Transparent Objects by Using Inverse Polarization Ray Tracing," IEEE Trans. On Pattern Analysis and Machine Intelligence, Vol. 29,pp. 2018-2030, (2007).

34. O. Morel, C. Stolz, F. Meriaudeau, P. Gorria, "Active Lighting Applied to 3D Reconstruction of Specular Metallic Surfaces by Polarization Imaging", Applied Optics, 45 (17), pp. 4062-4068, (2006). 
35. M. Ferraton, C. Stolz, F. Meriaudeau, "Optimization of a polarization imaging system for 3D measurements of transparent objects”, Optics Express, 17 (23), pp. 21077-21082, (2009).

36. S. J. Tyo, D. L. Goldstein, D. B. Chenault, and J. A. Shaw, "Review of passive imaging polarimetry for remote sensing applications," Appl. Opt. , Vol 45, pp. 5453-5469, (2006).

37. D. L. Goldstein, Polarized light second Edition (Optical Engineering), p. 680, (2003).

38. Rantoson R., Stolz C., Fofi D. and Mériaudeau F., '3D Reconstruction by polarimetric imaging method based on perspective model', Proc. SPIE Europe Optical Metrology, (2009). 